\title{
CHARITÉ
}

UNIVERSITÄTSMEDIZIN BERLIN

\section{Kostenevaluation eines Modells der Integrierten Versorgung für schwer psychisch Kranke}

Felix Fischer, Karin Hoffmann, Norbert Mönter, Matthias Walle, René Beneke, Sabine Negenborn, Stefan Weinmann, Stefan N. Willich, Anne Berghöfer

\section{Document type}

Postprint (accepted version)

This version is available at

https://doi.org/10.17169/refubium-33024

\section{Citation details}

Fischer F, Hoffmann K, Mönter N, Walle M, Beneke R, Negenborn S, et al. Kostenevaluation eines Modells der Integrierten Versorgung für schwer psychisch Kranke. Das Gesundheitswesen. Georg Thieme Verlag KG; 2013. p. 86-95. DOI: 10.1055/s-0033-1343438

\section{Terms of use}

All rights reserved. This document is intended solely for personal, non-commercial use. 


\section{Kostenevaluation eines Modells der Integrierten Versorgung für schwer psychisch Kranke}

\section{Cost Evaluation of a Model for Integrated Care of Seriously Mentally III Patients}

Felix Fischer ${ }^{1}$, Karin Hoffmann², Norbert Mönter ${ }^{3}$, Matthias Walle ${ }^{4}$, René Beneke ${ }^{5}$, Sabine Negenborn ${ }^{6}$, Stefan Weinmann ${ }^{1,7}$, Stefan N. Willich ${ }^{1}$, Anne Berghöfer ${ }^{1}$

${ }^{1}$ Institut für Sozialmedizin, Epidemiologie und Gesundheitsökonomie, Charité Universitätsmedizin Berlin

${ }^{2}$ Klinik und Poliklinik für Psychiatrie und Psychotherapie, Charité -

Universitätsmedizin Berlin

${ }^{3}$ Psychiatrie Initiative Berlin Brandenburg (PIBB) GmbH \& Co. KG und Verein für Psychiatrie und seelische Gesundheit (VPsG) e.V., Berlin

${ }^{4}$ Inno Health Care (IHC) GmbH \& Co. KG, Hemmoor und FIVP e.V., Niedersachsen ${ }^{5}$ DAK-Gesundheit, Versorgungsgebiet Niedersachsen/Bremen, Hannover ${ }^{6}$ DAK-Gesundheit, Versorgungsgebiet Berlin/Brandenburg, Berlin ${ }^{7}$ Deutsche Gesellschaft für Internationale Zusammenarbeit (GIZ)

\section{Korrespondenzadresse:}

Dr. med. Anne Berghöfer

Institut für Sozialmedizin, Epidemiologie und Gesundheitsökonomie

Charité - Universitätsmedizin Berlin

Luisenstr. 57

10117 Berlin

Tel.: 030/450529034

FAX 030/450529902

Email: anne.berghoefer@charite.de 


\section{Zusammenfassung}

Hintergrund: Das Modell der Integrierten Versorgung (IV) schwer psychisch Kranker für Versicherte der DAK-Gesundheit und verschiedener Betriebskrankenkassen (Mitglieder der BKK VAG Mitte) der Regionen Berlin, Brandenburg, Niedersachsen und Bremen ermöglicht eine ambulante Komplexbehandlung bestehend aus psychiatrischen Fachärzten, Haus- und Klinikärzten, psychiatrischer Fachpflege, Soziotherapie (nur Berlin), innerärztliche Qualitätszirkelarbeit, Orientierung an Behandlungspfaden und konzeptioneller Abstimmung mit den zuständigen Versorgungskliniken. Ziel der Evaluation war die Darstellung der gesundheitsökonomischen Auswirkungen der IV.

Methodik: Im Zeitraum zwischen 2006 und 2010 wurden Versicherte der DAKGesundheit und der beteiligten Betriebskrankenkassen mit einer schweren psychischen Erkrankung, einer deutlichen Beeinträchtigung des sozialen Funktionsniveaus und der Erfordernis einer krankenhausvermeidenden oder krankenhausersetzenden Behandlung in die IV eingeschlossen. Die Kostenperspektive war diejenige der Gesetzlichen Krankenversicherung. Für die gesundheitsökonomische Auswertung wurde die Inanspruchnahme über 18 Monate kontinuierliche IV-Behandlung mit den letzten 18 Monaten vor Einschluss in die IV verglichen. Der klinische Befund wurde während der IV quartalsweise mit globaler Schweregrad- (CGI) und Funktionsbeurteilung (GAF) erhoben.

Ergebnisse: Insgesamt wurden 1.364 Patienten mit IV-Behandlung in 66 Arztpraxen dokumentiert (davon 286 ICD-10 F2, 724 ICD-10 F32-F39). Das Durchschnittsalter betrug 48,8 Jahre, $69 \%$ waren weiblich. $24 \%$ verfügten über ein eigenes Einkommen, $40 \%$ bezogen Rente, die übrigen Patienten Transferleistungen verschiedener Art. In $54 \%$ der Fälle wurde IV klinikvermeidend, in $46 \%$ klinikersetzend eingesetzt. Der CGISchweregrad betrug bei Einschluss durchschnittlich 5,4, der GAF-Score 36,5. In die gesundheitsökonomische Analyse wurden die 226 Patienten mit kontinuierlicher Dokumentation über 18 Monate eingeschlossen. Die Anzahl der Krankenhaustage war in 18 Monaten während IV niedriger als in 18 Monaten vor IV (11,8 vs. 28,6 Tage, p < 0,001 ), die Ausgaben für stationäre Behandlung signifikant geringer (5.929 \pm 13.837 Euro vs. $2.458 \pm 6.940$ Euro, $p<0,001$ ), die Gesamtausgaben veränderten sich jedoch nicht signifikant (7.777 \pm 14.263 Euro vs. $7.321 \pm 7.910$ Euro, $p=0,65)$. Die erhebliche Einsparung bei den stationären Ausgaben wurde durch die Mehrausgaben bei 
Medikamenten und die Ausgaben der ambulanten Komplexleistungen kompensiert. Analoge Ergebnisse erbrachte die Analyse der 2 Subgruppen der Patienten mit Schizophrenie/ schizoaffektiver Störung $(n=66,40,9$ vs. 17,9 Tage, $p=0,03$; stationäre Ausgaben $9.009 \pm 15.677$ Euro vs. $3.650 \pm 8.486$ Euro, $p=0,02$; Gesamtausgaben $11.789 \pm 15.975$ Euro vs. $9.623 \pm 9.262$ Euro, $p=0,33$ ) und mit unipolarer Depression ( $n=90,29,8$ vs. 9,8 Tage, $p=0,006$; stationäre Ausgaben 5.664 \pm 14.921 Euro vs. $1.967 \pm 5.276$ Euro, $p=0,02$; Gesamtausgaben $7.146 \pm 15.164$ Euro vs. $6.234 \pm 6.292$ Euro, $p=0,57$ ).

Schlussfolgerung: Das IV-Modell konnte durch ein komplexes ambulantes Leistungsangebot die stationäre Inanspruchnahme deutlich senken und eine Gewichtsverlagerung in eine niedrigschwellige umfassende Versorgungsstruktur realisieren, ohne dass dies aus GKV-Perspektive mit einer Kostensteigerung verbunden war. Für eine detaillierte Darstellung klinischer Effekte sind weitere Studien erforderlich.

Schlüsselwörter: Integrierte Versorgung, ambulante psychiatrische Pflege, Soziotherapie, Kosten

\section{Abstract}

Background: The model for integrated care (IC) of the seriously mentally ill for those insured with the DAK-Gesundheit health insurance and various Betriebskrankenkassen (members of the VAG Mitte) from the regions Berlin, Brandenburg, Lower Saxony and Bremen allows a complex treatment in the outpatient setting which consists of psychiatrists, general practitioners and clinicians, psychiatric nursing, sociotherapy (only in Berlin), internal medicine quality circles, orientation on treatment guidelines and conceptual consensus with the relevant care clinics. The aim of the evaluation was to illustrate the health economic effects of IC.

Methods: In the period from 2006 to 2010 insured members of the DAK-Gesundheit and other involved health insurance companies with a serious mental illness, a significant impairment of social functioning and the need to be treated to avoid or substitute an in-clinic stay were included in the integrated care. The cost perspective was that of the statutory health insurance companies. For the health economic evaluation, the utilisation of continuous IC over 18 months was compared to the last 
18 months prior to the inclusion in IC. The clinical findings were gathered quarterly during the IC using CGI (Clinical Global Impressions) and GAF (Global Assessment of Functioning Scale).

Results: A total of 1,364 patients receiving IC in 66 doctor's practices were documented (of those, 286 had diagnoses of ICD-10 F2, 724 ICD-10 F32-F39). The median age was 48.8 years, $69 \%$ were female. $24 \%$ had their own source of income, $40 \%$ were on the pension, and the rest of the patients were receiving transfer benefits in some form. In $54 \%$ of the cases IC was used to avoid an in-hospital stay, in $46 \%$ of the cases to substitute an in-hospital stay. The degree of the CGI was 5.5 on average at the time of inclusion and the GAF score was 36.5 on average. The 226 patients with continuous documentation over 18 months were included in the health economic analysis. The number of days spent in hospital was lower during the IC period as compared to the 18 months prior to IV (11.8 vs. 28.6 days, $p<0.001)$, the inpatient costs were lower $(5,929 \pm 13,837$ Euro vs. $2,458 \pm 6,940$ Euro, $p<0.001)$, the total was not significantly changed $(7,777 \pm 14,263$ Euro vs. 7,321 $\pm 7,910$ Euro, $p=0.65)$. The substantial reduction of inpatient costs was compensated by the additional costs for medication and the costs of the complex outpatient care. Results were comparable for the 2 subgroups of schizophrenic/schizoaffective ( $n=66,40.9$ vs. 17.9 days, $p=$ 0.03 ; inpatient cost $9,009 \pm 15,677$ Euro vs. $3,650 \pm 8,486$ Euro, $p=0.02$; total expenditures 11,789 $\pm 15,975$ Euro vs. 9,623 $\pm 9,262$ Euro, $p=0.33$ ) and unipolar depressive patients ( $n=90,29.8$ vs. 9.8 days, $p=0.006$; inpatient cost $5,664 \pm 14,921$ Euro vs. 1,967 $\pm 5,276$ Euro, $p=0.02$; total expenditures 7,146 $\pm 15,164$ Euro vs. $6,234 \pm 6,292$ Euro, $p=0.57$ ).

Conclusion: The IC was able to considerably reduce the utilisation of inpatient treatment through offering a complex range of services in the outpatient setting and allowed for a weight-shift in a low-threshold comprehensive care structure without an increase in costs from the statutory health insurance companies' perspective. For a detailed description of clinical effects further studies are required.

Key words: integrated care, psychiatric nursing, sociotherapy, cost 


\section{Hintergrund}

Im fraktionierten psychiatrischen Versorgungssystem in Deutschland haben insbesondere psychisch Kranke mit intensiviertem Behandlungsbedarf erhebliche Probleme, eine kontinuierliche angemessene und umfassende medizinische Versorgung zu finden [1]. Häufig wechseln sie den behandelnden Arzt und müssen zum Teil mehrfach im Jahr, teils über längere Zeit, stationär behandelt werden, wenn krankheitsbedingt ein Zustand komplexen Hilfebedarfs gegeben ist. Die ambulanten Leistungserbringer sind in vielen Regionen nicht ausreichend ausgestattet und vernetzt, um ambulante Komplexleistungen für schwer psychisch Erkrankte anzubieten, wie dies beispielsweise psychiatrische Gesundheitszentren in Großbritannien oder auch Institutsambulanzen in Deutschland praktizieren [2]. Damit bleiben Qualitätsreserven im ambulanten Bereich sowie Synergien durch ein Zusammenwirken von ambulanten und stationären Leistungsanbietern ungenutzt, und viele Patienten erhalten eine ambulant unzureichende und nur in akuten Krisen zeitlich befristete (meist vollstationäre) Komplex-Behandlung. Vorwiegend in den USA und in Großbritannien wurden in den letzten 30 Jahren erfolgreich Behandlungsmodelle von collaborative und community care erprobt, die Elemente der Integrierten Versorgung und der Disease Management Programme des deutschen Gesundheitswesens beinhalten [3-5]. Neben einer Besserung des psychopathologischen Behandlungsergebnisses und der Lebensqualität konnten auch Kostensenkungen erreicht werden [6,7]. Seit Einführung des Gesundheitsmodernisierungsgesetzes in Deutschland, das mit dem $\S 140 \mathrm{ff}$ SGB $\vee$ auch für das psychiatrische Fachgebiet Chancen bot, die Defizite in der Versorgung psychisch Erkrankter zu reduzieren, besteht die Möglichkeit, mit Krankenkassen Verträge über eine sektorübergreifende Versorgung im Rahmen des Modellversuchs der Integrierten Versorgung nach § 140 a-d SGB V abzuschließen. In mehreren Regionen in Deutschland wurden daher Modelle integrierter Versorgung (IV) mit dem Schwerpunkt auf schweren psychischen Erkrankungen erprobt [8-10]. In Berlin, Brandenburg, Niedersachsen und Bremen wurde ein derartiges Programm eingeführt, welches die Anbieter therapeutischer Leistungen zu einer Versorgungsstruktur vernetzte [11-13]. Außerdem wurden in Berlin und Niedersachsen mit mehreren Kliniken intersektorale Verträge zur verbesserten Zusammenarbeit des ambulanten Versorgernetzes mit der stationären Psychiatrie abgeschlossen. Institutionsübergreifende Komplexleistungsprogramme wurden als Gemeinschaftsprogramm der therapeutischen Leistungsanbieter für 
Patienten der Krankenkassen DAK-Gesundheit (Vertragsgebiete Berlin/Brandenburg, Niedersachsen/Bremen) und teilnahmebereiten Betriebskrankenkassen (BKK Vertragsarbeitsgemeinschaft Mitte, ehemals VAG Ost) entwickelt. Diese wurden als integrierende Behandlungspfade angeboten und durch gemeinsame Qualitätszirkelarbeit auf ein Niveau gebracht, wie es den Leitlinien der psychiatrischen Fachverbände entspricht. Die freie Wahl des Leistungserbringers war für die Versicherten durch die Vereinbarung zur Integrierten Versorgung nicht eingeschränkt. Wesentliche Inhalte des Modells waren:

- Eine umfassende Feststellung des Hilfebedarfs der teilnehmenden Versicherten, um beurteilen zu können, wo dem Behandlungsbedarf durch Leistungen im Rahmen der pflichtgemäßen kassenärztlichen Sicherstellung entsprochen werden kann oder ob komplexe und intensivierte Behandlungsmaßnahmen nötig sind, die diesen Rahmen überschreiten. In diesem Falle wurde, soweit nicht aus medizinischen Gründen eine unmittelbare Klinikeinweisung geboten war, den Patienten Integrierte Versorgung angeboten.

- Die die Integrierte Versorgung akzeptierenden Patienten wurden soweit wie möglich ambulant behandelt oder innen ambulant ausgerichtete Behandlung vermittelt.

- Der rechtzeitige Einsatz rehabilitativer Maßnahmen und die flankierende Mitbeteiligung psychosozialer Leistungsanbieter sollten die Möglichkeiten zur Besserung der klinischen und psychosozialen Situation und zur Aktivierung der gesunden Anteile der Patienten ausschöpfen, um nachhaltig die Umstellung vom institutsbezogenen zum personenbezogenen Hilfeansatz zu realisieren.

- Ein komplexes Leistungsangebot mit besonderer Akzentsetzung im ambulanten Bereich wurde für die in die Integrierte Versorgung eingeschriebenen Patienten vorgehalten, um durch enge Vernetzung mit den Hausärzten, dem sozialpsychiatrischen Dienst und anderen Krisendiensten, niedergelassenen Psychotherapeuten, ambulanten Pflegediensten, sowie nur in Berlin zusätzlich Soziotherapie nach $\S 37 \mathrm{a}$ SGB $\vee$ eine Gewichtsverlagerung in eine niedrigschwellige umfassende Versorgungsstruktur zu erreichen.

Die Evaluation der Wirksamkeit und der Entwicklung der Ausgaben im IV-Programm aus GKV-Sicht erfolgte durch das Institut für Sozialmedizin, Epidemiologie und Gesundheitsökonomie, das externe Qualitätsmanagement des IV-Netzwerkes durch 
die Klinik und Poliklinik für Psychiatrie und Psychotherapie, Charité Universitätsmedizin Berlin.

Primäres Ziel der Evaluation war die Darstellung des Ressourcenverbrauchs der in die IV eingeschlossenen Patienten nach 18 Monaten im Vergleich zum 18Monatszeitraum vor Einschluss in die IV.

Folgende Erwartungen wurden formuliert:

1. Die Patienten in der IV-Gruppe haben im 18-Monats-Beobachtungszeitraum weniger stationäre Aufenthalte und eine kürzere Gesamt-Verweildauer im Vergleich zum 18-Monatszeitraum vor Einschluss in die IV

2. Die Gesamtausgaben der Krankenkassen im Zusammenhang mit psychiatrischer Erkrankung sind im 18-Monatszeitraum geringer als im 18Monatszeitraum vor IV

\section{Methodik}

Für die Evaluation wurde eine prospektive multizentrische nicht-vergleichende Beobachtungsstudie über 18 Monate durchgeführt. In Niedersachsen/Bremen wurden seit Beginn 2006 Patienten in die IV eingeschlossen, in Berlin seit Anfang 2007. Seitdem erfolgte kontinuierlich ein Einschluss von Patienten bis zum 31.12.2009 (DAKGesundheit, beide Vertragsgebiete, $n=1.243$ ) bzw. 31.3.2010 (Mitglieder der BKK VAG Mitte, nur Vertragsgebiet Berlin, $n=121$ ). Das Studiendesign ist in Abbildung 1 dargestellt.

Das Studienprotokoll sowie die Patienteninformationen, Einwilligungserklärungen und die Erfassungsinstrumente wurden von der Ethikkommission der Charité Universitätsmedizin Berlin genehmigt. Die Studie wurde in Übereinstimmung mit den ethischen Grundsätzen der Deklaration von Helsinki, in Erfüllung der ICH-GCPRichtlinien, und unter Berücksichtigung der Datenschutz-Vorschriften des Landes Berlin durchgeführt. 


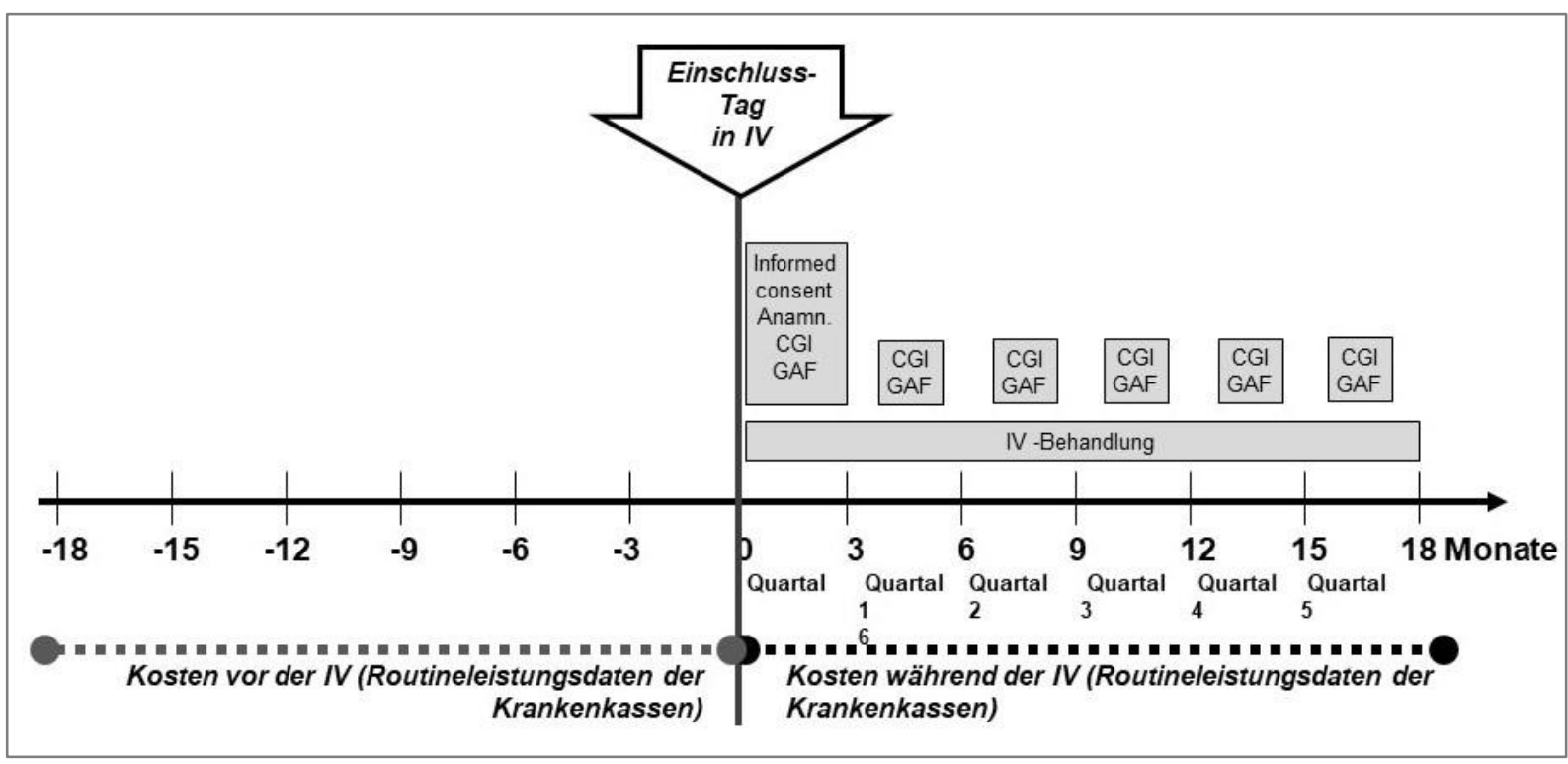

Abb. 1: Design der prä-/post-Vergleichsstudie von Patienten mit schweren psychischen Störungen in der Integrierten Versorgung.

Patienten, die in die Integrierte Versorgung eingeschlossen wurden, mussten folgende Kriterien erfüllen:

1. Vorliegen einer ICD-10 Diagnose aus dem Bereich F0.X bis F8.X

2. Erfordernis einer krankenhausvermeidenden Behandlung oder Notwendigkeit des Ersatzes einer stationären Behandlung durch ein komplexes Hilfeprogramm in der Einschätzung des behandelnden Arztes

3. Beeinträchtigungen des sozialen Funktionsniveaus (in der Global Assessment of Function Scale, GAF ein Score <= 50) [14]

4. globaler Schweregrad der Erkrankung von $>=5$ in der Clinical Global Impression Scale (CGI) [15]

5. Vorliegen der Einwilligungserklärung des Patienten und des gesetzlichen Betreuers, wenn der Patient gesetzlich betreut ist.

6. Alter mindestens 18 Jahre

Zusätzlich waren Schwellenwerte für krankheitsspezifische psychopathologische Skalen festgelegt.

Patienten, die folgende Kriterien erfüllten, konnten nicht in die IV eingeschlossen werden:

- Patienten, deren gesetzlicher Betreuer zugestimmt hat, die aber selbst nicht einwilligen möchten 
- Akute Suizidalität nach Einschätzung des behandelnden Arztes

- Hohe Wahrscheinlichkeit eines raschen Behandlungsabbruchs in der Einschätzung des behandelnden Arztes

$\mathrm{Da}$ das vorrangige Ziel des Ausbaus der ambulanten Komplexbehandlung die Reduktion stationärer Aufenthalte bei schwer psychisch Kranken und die Verlagerung der Versorgung in den ambulanten Sektor war, waren die Einschlusskriterien nicht diagnosespezifisch sondern in erster Linie an der Krankheitsschwere und der Indikation zur stationären Behandlung orientiert.

Der Einschluss von Patienten in die Integrierte Versorgung erfolgte kontinuierlich durch den behandelnden Psychiater/Nervenarzt. Die Erfassung der soziodemographischen Daten und der klinischen Daten erfolgte patientenindividuell durch die beteiligten Psychiater/Nervenärzte, bei denen die Patienten in psychiatrischer Behandlung waren. Die Erfassung der ökonomischen und Inanspruchnahmedaten erfolgte patientenindividuell durch Übermittlung von Abrechnungsdaten der beteiligten Krankenkassen.

Die Leistungen zur Soziotherapie (nur Berlin) und ambulanten psychiatrischen Pflege (APP) wurden mittels Abrechnungsdaten der Krankenkassen und der Leistungserbringer erfasst.

Bei Einschluss in die IV und in jedem weiteren Abrechnungsquartal wurden vom Behandler folgende Informationen dokumentiert:

- Soziodemographische Variablen (Psychiatrische Haupt- und Nebendiagnosen, somatische Diagnosen, Geschlecht, Wohnsituation, Vorrangiger Lebensunterhalt, Gesetzliche Betreuung, Alter bei Erstkrankung, Anzahl früherer stationärer Aufenthalte, Gesamtzahl Episoden/Schübe, Anzahl Suizidversuche)

- Aktuelle Eingruppierung nach Bedarf (klinikvermeidend, klinikersetzend)

- Bisherige Krankengeschichte

- Global Assessment of Function Scale (GAF) [14]

- Clinical Global Impression (CGI) [15]

Die Kostenanalyse basierte auf den Daten der Inanspruchnahme von Ressourcen im Rahmen der psychiatrischen Behandlung im ambulanten und stationären Sektor. Hierbei wurden Abrechnungsdaten der beteiligten gesetzlichen Krankenkassen verwendet. Die Kosten- (bzw. Ausgaben-)perspektive war diejenige der gesetzlichen Krankenversicherung (GKV). 
Berücksichtigt wurde lediglich diejenige Inanspruchnahme, die in den Zeitraum 18 Monate vor bis 18 Monate nach Ersteinschluss in die Integrierte Versorgung auftrat. Alle Inanspruchnahmekomponenten wurden auf Quartale umgerechnet, Kosten stationärer Aufenthalte wurden taggenau auf den Zeitraum vor oder nach Einschluss in die IV zugewiesen.

Für die ambulante Behandlung wurde pro Quartal die Kopfpauschale der GKV an die Kassenärztlichen Vereinigungen von 128 Euro angesetzt, zusätzlich wurde die IVVergütung von 130 Euro/Quartal für klinikvermeidende und 180 Euro/ Quartal für klinikersetzende Behandlung berücksichtigt. Hinzu kam eine Hausarztpauschale von 20 Euro/Quartal. Stationäre Aufenthalte und die Ausgaben für die Medikation wurden in Euro-Beträgen auf Basis der Routineleistungsdaten der GKV berücksichtigt, ambulante psychiatrische Pflege und Soziotherapie mit 48 Euro/h gemäß den Abrechnungen der Leistungserbringer angesetzt. Darüber hinaus entstanden pro Versicherten ein Kostenbeitrag von 10 Euro/Quartal für das externe Qualitätsmanagement in den Versorgungsnetzen sowie nur für Versicherte der DAKGesundheit Ausgaben von 10 Euro /Quartal für Erlass der Praxisgebühr.

In der statistischen Analyse wurden die Unterschiede zwischen durchgängig im IVProgramm behandelten Patienten und späteren „Drop-outs“ zu Baseline bei kategorialen Variablen (z. B. Diagnosehäufigkeit, Variablen zum Sozialstatus) mit ChiQuadrat- Tests und bei kontinuierlichen Variablen (z. B. Alter, Krankheitsschwere) mit $\mathrm{t}$-Tests auf statistische Signifikanz geprüft. Die Kostendaten der IV-Patienten im Beobachtungszeitraum von 18 Monaten wurden mit den Kostendaten des jeweiligen Patienten aus den letzten 18 Monaten vor Einschluss in das IV-Projekt verglichen. Der Vergleich der Mittelwerte erfolgte mittels t-Tests (bootstrapped) [16], weil die jeweilige Differenz der Kosten pro Datensatz zwischen dem Zeitraum vor IV und dem Zeitraum in IV nicht normalverteilt war. Als Statistik-Programm wurde $R$ in der Version 2.13 verwendet.

\section{Ergebnisse}

Im Zeitraum zwischen 2006 und 2010 wurden 1.364 Patienten in den Regionen Berlin, Brandenburg und Niedersachsen/Bremen in die IV eingeschlossen. Es nahmen 66 Arztpraxen teil, die zwischen einem und 79 IV-Patienten betreuten. Tabelle 1 zeigt die den Einschluss begründenden psychiatrischen Hauptdiagnosen. Die beiden größten 
Diagnosegruppen waren ICD-10 F2 (schizophrener Formenkreis, $\mathrm{n}=286$ ) und ICD-10 F32-F39 (Depression, $n=724$ ). Jeweils weniger als 10\% der Teilnehmer wurden wegen Angststörungen, Demenz oder Persönlichkeitsstörungen eingeschlossen. Eine oder mehrere psychiatrische Kodiagnosen wurden bei ca. der Hälfte der Patienten angegeben. Die soziodemografischen Charakteristika der Patienten bei Einschluss in die IV sind in Tabelle 2 dargestellt.

Tabelle 1: Hauptdiagnosen der eingeschlossenen Patienten nach ICD $10(N=1364)$ und der Subgruppe für die ökonomische Auswertung $(n=226)\left({ }^{*}\right.$ Chi-Quadrat-Test frühere Ausscheider vs. Subgruppe für ökonomische Auswertung).

\begin{tabular}{|c|c|c|c|}
\hline Diagnosen n (\%) & $\begin{array}{c}\text { Gesamt- } \\
\text { gruppe } \\
\mathrm{N}=1.364\end{array}$ & $\begin{array}{c}\text { Subgruppe in } \\
\text { ökonom. Analyse } \\
n=226\end{array}$ & $\mathrm{p}$-Wert ${ }^{\star}$ \\
\hline \multicolumn{4}{|l|}{ F0 organische psychische Störungen } \\
\hline F00-F03 Demenzen & $50(3,7)$ & $5(2,2)$ & 0,28 \\
\hline F06-F07 andere organische psychische Störungen & $15(1,1)$ & $1(0,4)$ & 0,49 \\
\hline F1 Störungen durch Missbrauch/Abhängigkeit & $21(1,5)$ & $4(1,8)$ & 0,99 \\
\hline \multicolumn{4}{|l|}{ F2 Schizophrenie und wahnhafte Störungen } \\
\hline F20 Schizophrenie & $190(13,9)$ & $51(22,6)$ & $<0,001$ \\
\hline $\begin{array}{l}\text { F21-F24, F29 andere akute und chron. } \\
\text { psychotische Störungen }\end{array}$ & $31(2,3)$ & $3(1,3)$ & 0,42 \\
\hline F25 schizoaffektive Erkrankung & $65(4,8)$ & $12(5,3)$ & 0,80 \\
\hline \multicolumn{4}{|l|}{ F3 Affektive Störungen } \\
\hline F30-31 bipolare Störung & $78(5,7)$ & $22(9,7)$ & 0,01 \\
\hline F32-F39 affektive Störung, Depression & $724(53,1)$ & $90(39,8)$ & $<0,001$ \\
\hline F4 Neurotische Störungen & $155(11,4)$ & $31(13,7)$ & 0,27 \\
\hline $\begin{array}{l}\text { F5, F6, F8 Persönlichkeitsstörungen u.a. } \\
\text { Störungen }\end{array}$ & $35(2,6)$ & $7(3,1)$ & 0,75 \\
\hline
\end{tabular}


Tabelle 2: Demografische und klinische Parameter der Gesamtgruppe ( $n=1364)$ und der Subgruppe für die ökonomische Auswertung $(n=226)$ bei Einschluss in die IV ( ${ }^{*}$ exakter Test nach Fisher/Chi-Quadrat, \# t-Test, jeweils frühere Ausscheider vs. Subgruppe für ökonomische Auswertung).

\begin{tabular}{|c|c|c|c|}
\hline & $\begin{array}{c}\text { Gesamt }(n=1.364) \\
n(\%)\end{array}$ & $\begin{array}{c}\text { Subgruppe in } \\
\text { ökonomischer Analyse } \\
(\mathrm{n}=226)\end{array}$ & p-Wert \\
\hline Alter (Jahre, MW, SD) & $48,8 \pm 16,0$ & $47,1 \pm 13,1$ & $0,554^{\#}$ \\
\hline Geschlecht weiblich, n (\%) & $939(68,8)$ & $161(71,2)$ & $0,391^{*}$ \\
\hline Lebensunterhalt & $n=1.210$ & $\mathrm{n}=\mathbf{2 0 1}$ & \\
\hline Eigenes Einkommen, n (\%) & $293(24,2)$ & $42(20,9)$ & $0,124^{*}$ \\
\hline Rente, n (\%) & $483(39,9)$ & $94(46,8)$ & \\
\hline Arbeitslosengeld, Krankengeld, $\mathrm{n}(\%)$ & $232(19,2)$ & $31(15,4)$ & \\
\hline Sozialhilfe, Arbeitslosenhilfe, $\mathrm{n}(\%)$ & $202(16,7)$ & $34(16,9)$ & \\
\hline Wohnsituation & $n=1.309$ & $\mathrm{n}=\mathbf{2 1 8}$ & \\
\hline Alleinwohnen in eigener Wohnung, $\mathrm{n}(\%)$ & $702(53,6)$ & $127(58,3)$ & $0,512^{*}$ \\
\hline $\begin{array}{l}\text { eigenständiges Wohnen mit Partner oder } \\
\text { Angehörigen, } \mathrm{n}(\%)\end{array}$ & $524(40,0)$ & $79(36,2)$ & \\
\hline Wohnen in elterlicher Wohnung, $\mathrm{n}(\%)$ & $58(4,4)$ & $7(3,2)$ & \\
\hline Wohnen im Heim/betreutes Wohnen, n (\%) & $25(1,9)$ & $5(2,3)$ & \\
\hline Gesetzliche Betreuung & $n=1.308$ & $n=219$ & \\
\hline vorhanden, n (\%) & $161(12,3)$ & $32(14,6)$ & $0,35^{\star}$ \\
\hline IV-Indikation & $n=1.303$ & $n=221$ & \\
\hline IV klinikvermeidend (n, \%) & $701(53,8)$ & $111(50,2)$ & $0,452^{*}$ \\
\hline IV klinikersetzend (n, \%) & $602(46,2)$ & $110(49,8)$ & \\
\hline \multicolumn{4}{|l|}{ Krankheitsschwere } \\
\hline Alter bei Ersterkrankung (Jahre, MW, SD) & $36,6 \pm 17,6$ & $35,5 \pm 14,3$ & $0,311^{\#}$ \\
\hline $\begin{array}{l}\text { Durchschn. Anzahl stationärer Voraufenthalte } \\
\text { vor IV }(\mathrm{n}, \mathrm{MW}, \mathrm{SD})\end{array}$ & $2,9 \pm 4,2$ & $4,1 \pm 5,5^{*}$ & $<0,001^{\#}$ \\
\hline $\begin{array}{l}\text { Durchschn. Anzahl Krankheitsepisoden einschl. } \\
\text { Indexepisode (n, MW, SD) }\end{array}$ & $4,6 \pm 6,8$ & $5,2 \pm 4,8^{*}$ & $0,027^{\#}$ \\
\hline CGI-Schweregrad (Score, MW, SD) & $5,4 \pm 0,7$ & $5,3 \pm 0,7$ & $0,187^{\#}$ \\
\hline GAF-Score (Score, MW, SD) & $36,5 \pm 8,8$ & $35,9 \pm 9,0$ & $0,259^{\#}$ \\
\hline
\end{tabular}

In die ökonomische Analyse über 18 Monate wurden 226 von 1.364 Patienten (215 Versicherte der DAK-Gesundheit, 11 Versicherte der BKKen Vertragsgebiet Berlin) eingeschlossen, somit alle, die über 6 Quartale kontinuierlich mit IV behandelt wurden. Diese Patienten hatten signifikant mehr Krankheitsepisoden vor IV (frühere Ausscheider MW 3,98 \pm 4,79, 18-Monats-Patienten MW 5,17 \pm 4,81, $p=0,027$ (t-Test)) und signifikant mehr stationäre Aufenthalte vor IV (frühe Ausscheider MW 2,31 $\pm 3,2$, 
18-Monats-Patienten MW 4,07 $\pm 5,53, p<0,001$ (t-Test), siehe Tab. 2), unterschieden sich in den übrigen Parametern jedoch nicht von der Gesamtgruppe.

Die Anzahl der Krankenhaustage konnte in der Zeit während der IV-Behandlung signifikant gegenüber dem Zeitraum vor IV gesenkt werden (Tab. 3). Die Gesamtausgaben für die psychiatrische Behandlung waren während der IV nicht signifikant gegenüber den Ausgaben in den 18 Monaten vor IV verändert. Die stationären Kosten waren während der IV zwar signifikant niedriger, dieser Vorteil wurde aber durch die höheren Arzneimittelausgaben sowie die zusätzlichen Ausgaben für die ambulante Komplexbehandlung verbraucht. Analoge Ergebnisse erbrachte die Analyse der 2 Subgruppen der Patienten mit Schizophrenie/ schizoaffektiver Störung $(n=66)$ und mit unipolarer Depression $(n=90)$. Auch hier sanken Anzahl der Krankenhaustage und stationäre Kosten signifikant.

Die Gesamtkosten waren in der Region Berlin/Brandenburg sowohl vor als auch während IV auf niedrigerem Niveau als in Niedersachsen/Bremen (Tab. 4), dort erfolgte hingegen eine stärkere Veränderung der Gesamtkosten. In Niedersachsen/Bremen entstanden höhere Ausgaben für ambulante psychiatrische Pflege als in Berlin/Brandenburg, da Soziotherapie dort nicht Bestandteil der ambulanten Komplexbehandlung war.

Der größte Teil der Patienten hatte vor IV niedrige Behandlungskosten zwischen 0 und 10.000 Euro in 18 Monaten. Die geringe Anzahl von Patienten mit sehr hohen Kosten (high utilizer; Behandlungskosten über 30.000 Euro bis zu 100.000 Euro) vor IV konnte während der IV deutlich kostengünstiger behandelt werden. Zugleich sind aber auch einige Patienten, für die vor IV wenig Ausgaben erforderlich waren, während der IV zu Hochnutzern bis zu 50.000 Euro über 18 Monate geworden (Abb. 2). 
Tabelle 3: Klinischer Schweregrad und GKV-Gesamtausgaben in Euro und Krankenhaustage über 18 Monate vor IV und 18 Monate während IV in Euro (SD, Median, Spannweite) über alle Diagnosen und stratifiziert nach großen Diagnosegruppen Schizophrenie/schizoaffektive Störung und unipolare Depression. Bei fixen Ausgabenpositionen wurde auf die Angabe von Median und Spannweite verzichtet.

\begin{tabular}{|c|c|c|c|c|c|c|c|c|c|}
\hline \multirow{2}{*}{$\begin{array}{l}\text { CGI bei IV-Einschluss } \\
\text { GAF bei IV-Einschluss } \\
\text { Ausgabenkomponente }\end{array}$} & \multicolumn{3}{|c|}{$\begin{array}{l}\text { Alle Diagnosen, } \mathbf{N}=\mathbf{2 2 6} \\
\qquad \begin{array}{l}5,3(0,7) \\
35,9(9,0)\end{array}\end{array}$} & \multicolumn{3}{|c|}{$\begin{array}{c}\text { Diagnose Schizophrenie/ schizoaffektive } \\
\text { Störung, } \mathbf{n}=66 \\
5,4(0,8) \\
35,5(8,1)\end{array}$} & \multicolumn{3}{|c|}{$\begin{array}{c}\text { Diagnose Depression, } \mathbf{n}=90 \\
\qquad \begin{array}{c}5,3(0,6) \\
36,0(9,0)\end{array}\end{array}$} \\
\hline & $\begin{array}{l}18 \text { Monate } \\
\text { vor IV }\end{array}$ & $\begin{array}{l}18 \text { Monate } \\
\text { in IV }\end{array}$ & p-Wert & $\begin{array}{l}18 \text { Monate } \\
\text { vor IV }\end{array}$ & $\begin{array}{l}18 \text { Monate } \\
\text { in IV }\end{array}$ & p-Wert & $\begin{array}{l}18 \text { Monate } \\
\text { vor IV }\end{array}$ & $\begin{array}{l}18 \text { Monate } \\
\text { in IV }\end{array}$ & p-Wert \\
\hline $\begin{array}{l}\text { Ausgaben für die amb. ärztlich- } \\
\text { psychiatrische Behandlung } \\
\text { (Kopfpauschale, IV-Vergütung) }\end{array}$ & $768(0)$ & $\begin{array}{l}1.658(147) \\
(1.648,768- \\
1.848)\end{array}$ & n.a. & $768(0)$ & $\begin{array}{l}1.625(153) \\
(1.598,768- \\
1.848)\end{array}$ & n.a. & $768(0)$ & $\begin{array}{c}1.668(124) \\
(1.648,1.418- \\
1.848)\end{array}$ & n.a. \\
\hline $\begin{array}{l}\text { Stationär-psychiatrische } \\
\text { Ausgaben }\end{array}$ & $\begin{array}{l}5.929(13.837) \\
(0,0-106.977)\end{array}$ & $\begin{array}{l}2.458(6.940) \\
(0,0-41.974)\end{array}$ & $<0,001$ & $\begin{array}{c}9.009(15.677) \\
(0,0-77.209)\end{array}$ & $\begin{array}{l}3.650(8.486) \\
(0,0-40.734)\end{array}$ & 0,02 & $\begin{array}{l}5.664(14.921) \\
(0,0-106.977)\end{array}$ & $\begin{array}{l}1.967(5.276) \\
(0,0-31.418)\end{array}$ & 0,02 \\
\hline Krankenhaustage & $\begin{array}{l}28,6(66,8) \\
(0,0-456)\end{array}$ & $\begin{array}{l}11,8(33,2) \\
(0,0-192)\end{array}$ & $<0,001$ & $\begin{array}{l}40,9(73,9) \\
(0,0-401)\end{array}$ & $\begin{array}{l}17,9(41,8) \\
(0,0-187)\end{array}$ & 0,030 & $\begin{array}{l}29,8(73,9) \\
(0,0-456)\end{array}$ & $\begin{array}{l}9,8(26,4) \\
(0,0-166)\end{array}$ & 0,006 \\
\hline $\begin{array}{l}\text { Ausgaben für } \\
\text { Rehabilitationsaufenthalte }\end{array}$ & $\begin{array}{c}72(625) \\
(0,0-7.427)\end{array}$ & $\begin{array}{c}68(536) \\
(0,0-5.740)\end{array}$ & 0,95 & 0 & $\begin{array}{c}57(461) \\
(0,0-3.742)\end{array}$ & n.a. & $\begin{array}{c}171(982) \\
(0,0-7.427)\end{array}$ & $\begin{array}{c}62(453) \\
(0,0-4.005)\end{array}$ & 0,35 \\
\hline $\begin{array}{l}\text { Ausgaben für psychiatrische } \\
\text { Medikation }\end{array}$ & $\begin{array}{c}1.008(1.633) \\
(293,0-10.771)\end{array}$ & $\begin{array}{c}1.548(2.153) \\
(540,0-12.384)\end{array}$ & $<0,001$ & $\begin{array}{c}2.012(2.290) \\
(1.332,0- \\
10.771)\end{array}$ & $\begin{array}{c}2.797(2.897) \\
(1.878,0-12.384)\end{array}$ & $<0,01$ & $\begin{array}{c}542(891) \\
(218,0-4.817)\end{array}$ & $\begin{array}{r}1.021(1.487) \\
(463,0-8.235)\end{array}$ & $<0,01$ \\
\hline $\begin{array}{l}\text { Ausgaben für ambulante } \\
\text { psychiatrische Pflege }\end{array}$ & - & $\begin{array}{c}865(1.726) \\
(147,0-14.124)\end{array}$ & n.a. & - & $\begin{array}{l}637(1.714) \\
(0,0-12.704)\end{array}$ & n.a. & - & $\begin{array}{c}849(1.063) \\
(483,0-4.824)\end{array}$ & n.a. \\
\hline $\begin{array}{l}\text { Ausgaben für ambulante } \\
\text { Soziotherapie (nur in Berlin) }\end{array}$ & - & $\begin{array}{c}488(895) \\
(0,0-3.648)\end{array}$ & n.a. & - & $\begin{array}{l}624(1.022) \\
(0,0-3.528)\end{array}$ & n.a. & - & $\begin{array}{c}430(810) \\
(0,0-3.289)\end{array}$ & n.a. \\
\hline Hausarztpauschale & - & $120(0)$ & n.a. & - & $120(0)$ & n.a. & - & $120(0)$ & n.a. \\
\hline Qualitätsmanagement & - & $60(0)$ & n.a. & - & $60(0)$ & n.a. & - & $60(0)$ & n.a. \\
\hline Erlass Praxisgebühr (DAK-Pat.) & - & $\begin{array}{c}56(15) \\
(60,0-60)\end{array}$ & n.a. & - & $60(0)$ & n.a. & - & $\begin{array}{c}57(12) \\
(60,0-60)\end{array}$ & n.a. \\
\hline Gesamtausgaben & $\begin{array}{c}7.777(14.263) \\
(1.554 \\
768-109.316)\end{array}$ & $\begin{array}{c}7.321(7.910) \\
(4.456, \\
1.269-51.017)\end{array}$ & 0,65 & $\begin{array}{c}11.789(15.975) \\
(4.799 \\
768-78.906)\end{array}$ & $\begin{array}{c}9.623(9.262) \\
(5.978, \\
1.728-48.034)\end{array}$ & 0,33 & $\begin{array}{c}7.146(15.164) \\
(1.183, \\
768-109.317)\end{array}$ & $\begin{array}{c}6.234(6.292) \\
(4.287 \\
1.976-39.015)\end{array}$ & 0,57 \\
\hline
\end{tabular}


Tabelle 4: Klinischer Schweregrad und GKV-Gesamtausgaben in Euro und Krankenhaustage über 18 Monate vor IV und 18 Monate während IV in Euro (SD, Median, Spannweite) stratifiziert nach Versorgungsregionen. Bei fixen Ausgabenpositionen wurde auf die Angabe von Median und Spannweite verzichtet.

\begin{tabular}{|c|c|c|c|c|c|c|}
\hline \multirow{2}{*}{$\begin{array}{l}\text { CGI bei IV-Einschluss } \\
\text { GAF bei IV-Einschluss } \\
\text { Ausgabenkomponente }\end{array}$} & \multicolumn{3}{|c|}{$\begin{array}{c}\text { Region Niedersachsen/Bremen, } \mathbf{n}=\mathbf{9 7} \\
5,2(0,7) \\
37,5(9,1)\end{array}$} & \multicolumn{3}{|c|}{$\begin{array}{c}\text { Region Berlin/Brandenburg, } \mathbf{n}=\mathbf{1 2 9} \\
5,4(0,7) \\
34,7(8,7)\end{array}$} \\
\hline & $\begin{array}{l}18 \text { Monate } \\
\text { vor IV }\end{array}$ & $\begin{array}{l}18 \text { Monate } \\
\text { in IV }\end{array}$ & p-Wert & $\begin{array}{l}18 \text { Monate } \\
\text { vor IV }\end{array}$ & $\begin{array}{l}18 \text { Monate } \\
\text { in IV }\end{array}$ & p-Wert \\
\hline $\begin{array}{l}\text { Ausgaben für die amb. ärztlich- } \\
\text { psychiatrische Behandlung } \\
\text { (Kopfpauschale, IV-Vergütung) }\end{array}$ & $768(0)$ & $\begin{array}{c}1.646(166) \\
(1.648,764-1.848)\end{array}$ & n.a. & $768(0)$ & $\begin{array}{c}1.666(130) \\
(1648,1418-1848)\end{array}$ & n.a. \\
\hline $\begin{array}{l}\text { Stationär-psychiatrische } \\
\text { Ausgaben }\end{array}$ & $\begin{array}{l}6.892(17.126) \\
(0,0-106977)\end{array}$ & $\begin{array}{l}2.878(7.354) \\
(0,0-41975) \\
\end{array}$ & 0,03 & $\begin{array}{c}5.206(10.739) \\
(0,0-55434) \\
\end{array}$ & $\begin{array}{l}2.143(6.622) \\
(0,0-40734) \\
\end{array}$ & $<0,001$ \\
\hline Krankenhaustage & $\begin{array}{c}33,4(81,9) \\
(0,0-456)\end{array}$ & $\begin{array}{c}13,8(36,9) \\
(0,0-192)\end{array}$ & 0,025 & $\begin{array}{c}25,0(52,8) \\
(0,0-266)\end{array}$ & $\begin{array}{l}10,2(30,2) \\
(0,0-166)\end{array}$ & 0,003 \\
\hline $\begin{array}{l}\text { Ausgaben für } \\
\text { Rehabilitationsaufenthalte }\end{array}$ & $\begin{array}{c}41(333) \\
(0,0-3184)\end{array}$ & $\begin{array}{c}156(812) \\
(0,0-5740)\end{array}$ & 0,21 & $\begin{array}{c}95(776) \\
(0,0-7427)\end{array}$ & $\begin{array}{c}3(28) \\
(0,0-322)\end{array}$ & 0,18 \\
\hline $\begin{array}{l}\text { Ausgaben für psychiatrische } \\
\text { Medikation }\end{array}$ & $\begin{array}{c}1.092(1.712) \\
(326,0-9493)\end{array}$ & $\begin{array}{c}1.767(2.361) \\
(689,0-12384)\end{array}$ & 0,0 & $\begin{array}{c}944(1.574) \\
(275,0-10771)\end{array}$ & $\begin{array}{c}1.383(1.976) \\
(503,0-11633)\end{array}$ & $<0,001$ \\
\hline $\begin{array}{l}\text { Ausgaben für ambulante } \\
\text { psychiatrische Pflege }\end{array}$ & - & $\begin{array}{c}1.233(1.801) \\
(684,0-12704)\end{array}$ & n.a. & - & $\begin{array}{l}589(1.619) \\
(0,0-14124) \\
\end{array}$ & n.a. \\
\hline $\begin{array}{l}\text { Ausgaben für ambulante } \\
\text { Soziotherapie (nur in Berlin) }\end{array}$ & - & - & n.a. & - & $\begin{array}{l}855(1.045) \\
(0,0-3648) \\
\end{array}$ & n.a. \\
\hline Hausarztpauschale & - & $120(0)$ & n.a. & - & $120(0)$ & n.a. \\
\hline Qualitätsmanagement & - & $60(0)$ & n.a. & - & $60(0)$ & n.a. \\
\hline Erlass Praxisgebühr (DAK-Pat.) & - & $60(0)$ & n.a. & - & $\begin{array}{c}53(20) \\
(60,0-60) \\
\end{array}$ & n.a. \\
\hline Gesamtausgaben & $\begin{array}{c}8.794(17.375) \\
(1848,768-109.317)\end{array}$ & $\begin{array}{c}7.919(8.638) \\
(4.348,1.269-51.017)\end{array}$ & 0,63 & $\begin{array}{c}7.013(11.394) \\
(1.395,768-57.028)\end{array}$ & $\begin{array}{c}6.871(7.318) \\
(4.472,1.728-48.034)\end{array}$ & 0,89 \\
\hline
\end{tabular}




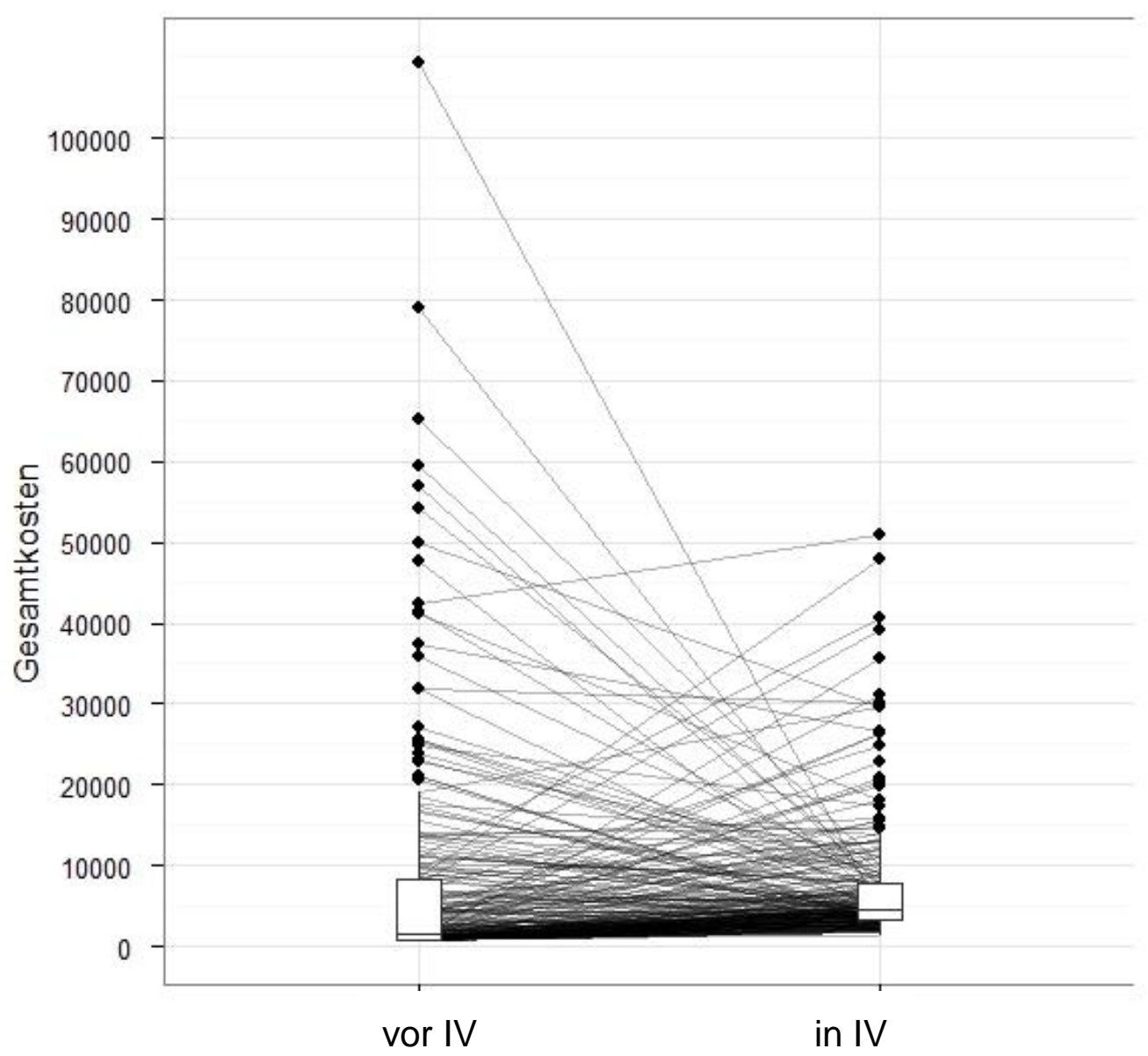

Abbildung 2: Darstellung der individuellen Kostenentwicklung von 226 Patienten je 18 Monate vor und in IV. Jeder Patient wird durch eine Markierung auf der linken Säule mit seinen Kosten vor IV und mit einer Markierung auf der rechten Säule mit seinen Kosten in IV dargestellt, die Verbindungslinie stellt dar, ob die Kosten dieses Patienten abgenommen oder zugenommen haben.

\section{Diskussion}

Das evaluierte Modell der Integrierten Versorgung bei schweren psychischen Erkrankungen konnte die Anzahl der Krankenhaustage und stationären Ausgaben in einem Versorgungszeitraum von 18 Monaten signifikant gegenüber dem Zeitraum von 18 Monaten vor IV senken. Die Gesamtausgaben aus Sicht der gesetzlichen Krankenversicherung veränderten sich nicht signifikant. Bei hoher Kostenvarianz ist die Veränderung für die Gesamtgruppe statistisch nicht signifikant. Unter der IVBehandlung verringerte sich jedoch die Standardabweichung, weil Kostenausreißer nur bis zu einem halb so hohen Betrag auftraten. Die Arzneimittelausgaben stiegen unter IV Behandlung signifikant an. Dies ist darauf zurückzuführen, dass bei Reduktion 
der stationären Behandlungszeit die ambulante Behandlungszeit zugleich ansteigt. Während in den stationären Kosten die Arzneimittel aus Perspektive der GKV bereits enthalten sind, fallen im ambulanten Sektor die Arzneimittelausgaben nun gesondert an. Eine weitere Erklärungsmöglichkeit ist eine Zunahme leitlinienorientierter Pharmakotherapie bzw. eine häufigere Verwendung neuerer und teurerer Antipsychotika und Antidepressiva im Rahmen des Qualitätsmanagements ambulanter Prozesse während der IV.

Die Anzahl der Krankenhaustage sowie die Gesamtausgabe lagen in Niedersachsen/Bremen vor IV höher als in Berlin/Brandenburg. Beide Regionen konnten die Anzahl stationärer Tage unter IV-Behandlung signifikant senken. Die unterschiedlichen Ergebnisse in den beiden Regionen können durch Unterschiede in der Versorgungsstruktur zwischen ländlicher und städtischer Region erklärt werden. Im Flächenland Niedersachsen bestanden größere Distanzen und ein schwächeres Netz an ambulanten Angeboten mit der Folge, dass Entlassungen erst bei höherer Stabiliät des Krankheitsbildes möglich waren. Die Region Berlin hat dagegen in einigen Bezirken eine jahrzehntelange sozialpsychiatrische Tradition, die wegen der räumlichen Nähe im städtischen Raum und des universitären Einflusses bereits früh zu einer Arbeit in Netzwerken unter Integration komplementärer Leistungsanbieter führte. Faktisch ist bereits lange vor Beginn des IV-Modells stellenweise eine Behandlung chronisch kranker Patienten nach einem Ansatz erfolgt, der dem IVModell nahekommt. Dies könnte zum Teil die niedrigere Ausgangslage in den Gesamtausgaben und den Krankenhaustagen in der Region Berlin erklären.

Die Ausgaben für die APP sind in Niedersachsen möglicherweise höher, da sie stellenweise Ersatzfunktion für die nicht angebotene Soziotherapie übernehmen musste. Während die ambulante psychiatrische Fachpflege ein versorgendes und fürsorgliches Leistungsangebot ist, zielt die Soziotherapie auf Förderung der Autonomie des Patienten ab und ist damit im Rahmen des komplexen ambulanten Leistungsangebotes ein differenzialtherapeutischer Gewinn.

Von den in der psychiatrischen Versorgung in Deutschland existierenden IV-Modellen wurden nur sehr wenige bisher im Hinblick auf ökonomische Fragestellungen wissenschaftlich untersucht. Hierzu gehört das "Hamburger Modell“ am Universitätsklinikum Eppendorf (UKE), welches sich durch die Einbindung aufwändiger gemeindepsychiatrischer Elemente auszeichnet $[17,18]$ und als einziges IV-Modell im Rahmen einer kontrollierten prospektiven Studie evaluiert wurde [19]. 
Auch hier war IV mit einer signifikanten Ausgabensenkung im stationären Sektor, jedoch einer Ausgabensteigerung im ambulanten Sektor verbunden, die letztlich in einer nicht signifikanten Veränderung der Gesamtausgaben resultierte. Die Ausgaben von ca. 15500 Euro/Jahr in der Standardbehandlung und ca. 13000 Euro/Jahr in der integrierten Behandlung sind mit den Ergebnissen unserer Evaluation nicht vergleichbar, da es sich in Hamburg um ein selektiertes schwerkrankes Klientel von Patienten mit Schizophrenie handelte [20].

Evaluationsergebnisse aus dem Münchner Modell des BKK Landesverband, Klinikum Rechts der Isar und der Arbeitsgemeinschaft Münchner Nervenärzte und Psychiater e.V. für Patienten mit Schizophrenie oder affektiven Störungen zeigten eine Reduktion stationärer Behandlungskosten um ca. $50 \%$ infolge eines integrierten ambulanten Programms zur Complianceförderung [21], eine ausführliche Publikation der Ausgabenanteile liegt hierzu jedoch nicht vor.

Sowohl das Münchner Modell als auch das hier dargestellte IV-Modell weisen keine besonders ausgeprägte Integration auf, weder im Sinne der Anzahl integrierter Sektoren, noch im Ausmaß der finanziellen Verantwortungsübernahme, noch im Sinne einer populationsbezogen umfassenden Versorgung. Ein diesbezüglich sehr stark integriert arbeitendes Modell ist das des Regionalen Psychiatrie Budgets (RPB), bei dem einem stationären Leistungsanbieter als Vertragspartner mit dem regionalen Verbund der Krankenkassen ein festes Jahresbudget für die gesamte regionale psychiatrische Versorgung bereitgestellt wird, innerhalb dessen der Vertragspartner sektorenübergreifend die Versorgung integriert gestalten kann. Eine Evaluation konnte hier eine langfristige Kostenstabilisierung nachweisen, jedoch ebenfalls keine signifikante Senkung der Gesamtkosten [22,23].

Die wenigen gesundheitsökonomischen Evaluationen von IV-Modellen in Deutschland zeigen keine signifikante Reduktion der Gesamtausgaben, anders als umfangreiche Daten aus innovativen Versorgungsmodellen für psychisch Kranke in den USA und England nahelegen [7]. Eine der am umfangreichsten gesundheitsökonomisch untersuchten Integrationsformen ist das Modell des collaborative care bei Depression, bestehend aus hausärztlich-fachärztlicher Kooperation, case management und algorithmusgestützten Behandlungspfaden [24], welches aber keine schwer psychisch Kranken im fachärztlichen Versorgungsbereich einschließt. Eine auf gemeindepsychiatrische Elemente bauende integrierte Behandlung schwer psychisch 
Kranker zeigte sich hingegen in einer US-amerikanischen Studie nicht kosteneffektiver [25] gegenüber einer Standardbehandlung.

Die vorliegende Evaluation ist durch mehrere Limitationen gekennzeichnet. Erstens konnten die ambulante Hauskrankenpflege und ambulanter Soziotherapie vor Einschluss in die IV nicht einbezogen werden, da diese Ausgaben der Krankenkasse nicht als Abrechnungsdaten vorlagen bzw. psychiatrischen Diagnosen nicht zugeschlüsselt werden konnten. Damit lag eine Unterschätzung der ambulanten psychiatrischen Krankenpflege und Soziotherapie vor Einschluss in die IV vor, deren Höhe nicht genau beziffert werden kann. Diese Limitation führte allenfalls zu einer Unterschätzung der Gesamtausgaben vor IV und damit zu einer Unterschätzung eines möglichen Einspareffekts durch die IV.

Zweitens musste sich die ökonomische Auswertung auf diejenigen Patienten beschränken, für die Dokumentationsbögen der Ärzte kontinuierlich vorlagen. Patienten, deren Gesundheitszustand sich deutlich verbesserte und die keinen Anspruch auf weitere IV hatten, oder solche, die abbrachen oder bei denen der Psychiater keinen Dokumentationsbogen übersandte, konnten nicht eingeschlossen werden, was zu einer gewissen Selektion von Nonrespondern in der Auswertungspopulation führte.

Drittens ist auch ein Rater-Bias durch Ärzte möglich, die Patienten in den psychopathologischen Skalen kränker einschätzten, obwohl sich ihr psychopathologischer Zustand verbesserte, um sie weiter in der IV zu halten. Diese mit der Vertragsstruktur innerhalb der IV verbundene Einschränkung könnte nur mit einer Datenerhebung durch unabhängige Untersucher vermieden werden, was im Rahmen des Evaluationsvertrages nicht zur Verfügung stand, aber bei späterer Begleitforschung eingesetzt werden sollte.

Viertens ist der methodische Ansatz eines Prä-Post-Vergleichs ohne Kontrollgruppe einschränkend, da unklar bleibt, wie sich der Ressourcenverbrauch der eingeschriebenen Patienten ohne die Intervention einer IV entwickelt hätte. Dies hängt in erster Linie davon ab, in welchem Krankheitsstadium die Patienten rekrutiert wurden, und wie die sonstige Ausgestaltung des Versorgungssystems vor IV war. Die hier eingeschlossenen Patienten wurden zum Teil in einer Krisensituation eingeschrieben, die normalerweise einen Krankenhausaufenthalt induzieren würde. Dies bedingt in jedem Fall zunächst höhere Kosten der Versorgung im Vergleich zur Situation vor der Krise (Exazerbation). Insbesondere für Ersterkrankte ist ein Prä-Post- 
Vergleich daher ungeeignet. Ersterkrankte wurden allerdings in dieses Modell wenig eingeschlossen.

Diese gesundheitsökonomische Auswertung erstreckt sich nur über 18 Monate. Die gesundheitsökonomischen Aussagen zu diesem IV-Modell, das langfristig angelegt ist, und bei dem bei etlichen Patienten erst in der längeren Frist deutliche Effekte hinsichtlich des Verlaufs und der Notwendigkeit stationär-psychiatrischer Behandlung erreicht werden, müssen daher vor dem Hintergrund dieses vergleichsweise kurzen Zeitraums betrachtet werden.

Die Quartalssichtweise führte zu einer Reduktion der Präzision der Kostendarstellung. Stationäre Kosten wurden jedoch taggenau dem Zeitraum vor bzw. in IV zugeordnet. Die Quartalssichtweise wurde im Hinblick auf die Machbarkeit der Dokumentation für die Leistungserbringer gewählt. Eine Stichtag-bezogene Analyse hätte eine exakte Terminkoordination in den Praxen erfordert, denn jeder Patient hätte Follow-upTermine in einem bestimmten Zeitfenster einhalten müssen. Um den Patienteneinschluss nicht mit Hürden, wie sie aus klinischen Studien bekannt sind, zu behindern, und damit das IV-Modell als Behandlungsmodell grundsätzlich zu gefährden, wurde dieser Kompromiss im Hinblick auf die Datenanalyse geschlossen. Die Integration von Routineleistungsdaten der Krankenkassen erwies sich als insgesamt noch aufwändig und komplex. Die Kopplung an die in der Evaluation erhobenen Daten bedurfte erheblicher händischer Bearbeitung und Kontrolle, um sie den Daten der IV-Dokumentation korrekt zuzuordnen und dabei Datenschutzauflagen nicht zu verletzen.

Im Hinblick auf die Kosten für die Evaluation kamen die Vertragspartner überein, auf ein Monitoring der Praxen, wie es in klinischen Studien erfolgt, zu verzichten. Die Datenqualität wurde durch Vollkontrolle der Dokumentation und Einfordern fehlender Angaben bei den Leistungserbringern gesichert, jedoch nicht durch Abgleich mit Originaldaten in der jeweiligen Arztpraxis. Eine Mitarbeit der Praxen im Qualitätsmanagement war vertraglich vorgesehen, der Umfang der Teilnahme variierte jedoch erheblich.

Schließlich war die Kostenperspektive für diese gesundheitsökonomische Auswertung diejenige der gesetzlichen Krankenversicherung. Ausgaben anderer Sozialversicherungsbereiche und indirekte Kosten wurden nicht mit einbezogen. Fallbezogene Ausgaben über alle SGB-Bereiche sind im deutschen Gesundheitswesen bislang nicht analog zu den Routineleistungsdaten der GKV 
verfügbar, sie wären mit begrenzter Aussagekraft nur über Auskünfte des Patienten und seiner Angehörigen erhebbar.

\section{Schlussfolgerungen}

Die gesundheitsökonomische Auswertung zeigte, dass das evaluierte IV-Modell insgesamt ein ökonomisch sinnvolles Modell war, mit dem eine signifikante Reduktion stationärer Behandlungskosten erreicht werden konnte. Die ursprüngliche Erwartung, dass die Gesamtkosten unter IV niedriger sind als vor IV, konnte nicht bestätigt werden, da die Einsparungseffekte infolge der Verringerung der stationären Behandlungskosten durch Mehrausgaben in anderen Bereichen (u. a. Ausgaben für Medikamente im ambulanten Bereich sowie Modellfinanzierung) kompensiert wurden. Allerdings trat unter IV auch über einen längeren Zeitraum keine Kostensteigerung ein. Das Modell würde in Zukunft erheblich von flächendeckend stärkerer Kooperation mit stationären Leistungserbringern profitieren. In beiden Regionen wurden inzwischen entsprechende Kooperationsverträge mit Krankenhäusern abgeschlossen. Auch ein Einbezug der Leistungserbringer in die unmittelbare Kostenverantwortung wäre als sinnvolle Modifikation anzusehen, insofern, als seitens des Arztes, der das Case Management übernommen hat, dann ein Anreiz zur optimalen und kurzfristigen Gestaltung des ambulanten Komplexangebots mit Verzahnung des stationären Sektors entsteht. Die Einschreibung von Patienten in die IV sollte zukünftig auf einen längeren Zeitraum ausgelegt sein, während dessen der Patient unabhängig vom individuellen Krankheitsverlauf eingeschrieben bleibt; dies wurde teilweise inzwischen umgesetzt. Das hier evaluierte Modell der Integrierten Versorgung schwer psychisch Kranker ist in einzigartiger Zusammenarbeit von mehreren Krankenkassen, den Leistungserbringern mehrerer Regionen in Deutschland und einer unabhängigen Evaluationsstelle entwickelt worden. Von den Leistungserbringern wurde hierzu über Jahre umfangreich in die Verbesserung der Strukturqualität investiert. Das Modell wird von den Akteuren als ein „lernendes Modell“ angesehen, welches auf der Basis von Evaluationsergebnissen Struktur- und Prozessoptimierung vornehmen will.

Interessenkonflikt: Die Autoren geben an, dass kein Interessenkonflikt besteht. 


\section{Literatur}

${ }^{1}$ Becker T, Hoffmann H, Puschner B, Weinmann S. Versorgungsmodelle in Psychiatrie und Psychotherapie. Stuttgart: Kohlhammer; 2008

2 Thornicroft G, Tansella M. The Mental Health Matrix. A Manual to Improve Services. Cambridge: Cambridge University Press; 2007

3 Gilbody S, Bower P, Fletcher J, Richards D, Sutton AJ. Collaborative care for depression. A cumulative meta-analysis and review of longer-term outcomes. Arch Intern Med 2006a;166;2314-2321.

${ }^{4}$ Mueser KT, Bond GR, Drake RE, Resnick SG. Models of community care for severe mental illness: a review of research on case management. Schizophr Bull $1998 ; 24(1) ; 37-74$

${ }^{5}$ Burns T, Catty J, Dash M, Roberts C, Lockwood A, Marshall M. Use of intensive case management to reduce time in hospital in people with severe mental illness: systematic review and meta-regression. Br Med J 2007; 335(7615):336

${ }^{6}$ Dieterich M, Irving CB, Park B, Marshall M. Intensive case management for severe mental illness. The Cochrane Library 2011; Vol. 2.

7 Gilbody S, Bower P, Whitty P. Costs and consequences of enhanced primary care for depression. Systematic review of randomised economic evaluations. $\mathrm{Br} \mathrm{J}$ Psychiatr 2006b;189;297-308.

8 Amelung VE, Meyer-Lutterloh K, Schmid E, Seiler R, Lägel R, Weatherly R: Integrierte Versorgung und Medizinische Versorgungszentren. 2. Auflage mit CDROM, Medizinisch Wissenschaftliche Verlagsgesellschaft, Berlin 2008

${ }^{9}$ Amelung VE, Bergmann F, Falkai P, Hauth I, Jaleel P, Meier U, Reichmann H, RothSackenheim C (Hrsg.). Innovative Konzepte im Versorgungsmanagement von ZNSPatienten. Berlin: Medizinisch Wissenschaftliche Verlagsgesellschaft, 2010

10 Grothaus F-J. Entwicklung der integrierten Versorgung in der Bundesrepublik Deutschland 2004 - 2008. Bericht gemäß § 140d SGB V auf der Grundlage der Meldungen von Verträgen zur integrierten Versorgung. Gemeinsame Registrierungsstelle zur Unterstützung der Umsetzung des § 140d SGB V. 2009

11. Walle $M$, Lammers-Reißing $A$, Munzel $\mathrm{H}$ et al. Integrierte Versorgung für psychisch schwer und chronisch Kranke am Beispiel Hemmoor und Weyhe/Niedersachsen. Nervenarzt 2005; 76 : $1030-1035$ 
12. Walle M, Reichwaldt W. Durch verbesserte Vernetzung zu mehr Qualität und Wirtschaftlichkeit. Integrierte Versorgung im gemeindepsychiarischen Kontext - das niedersächsische Modell. Soziale Psychiatrie 2010; 34: 22 - 25

13. Meyer G, Mönter N, Scheytt D. Kommunikation - Vertrauen - Integration. Der Berliner Verein für Psychiatrie und seelische Gesundheit e.V. und die Aufbaustufen einer sektorübergreifenden integrierten Versorgung. Soziale Psychiatrie 2010; 34: $46-49$

14. Wittchen HU et al. (Hrsg). Diagnostische Kriterien und Differentialdiagnosen des diagnostischen und statistischen Manuals psychischer Störungen DSM-IIIR. Weinheim, Basel: Beltz; 1989

15. Weyer G. Internationale Skalen für Psychiatrie. Göttingen: Beltz-Test; 2005

16. Efron B, Tibshirani R. An introduction to the bootstrap. New York: Chapman; 1993

17. Ohm G, Lambert M, Weatherly J N. Assertive Community Treatment psychotischer Patienten. In: Weatherly JN, Lägel R, Hrsg. Neue Versorgungsansätze in der Psychiatrie, Neurologie und Psychosomatik. Berlin: Medizinisch Wissenschaftliche Verlagsgesellschaft; 2009; 183 - 189

18. Lambert M, Naber D, Bock $T$ et al. Integrierte Versorgung von Patienten mit psychotischen Erkrankungen: das Hamburger Modell. In: Amelung V E, Bergmann F, Falkai P, Hauth I, Jaleel P, Meier U, Reichmann H, Roth-Sackenheim C, Hrsg. Innovative Konzepte im Versorgungsmanagement von ZNS-Patienten. Berlin: Medizinisch Wissenschaftliche Verlagsgesellschaft; 2010; 113 - 137

19. Lambert M, Bock T, Schöttle D et al. Assertive community treatment as part of integrated care versus standard care: a 12-month trial in patients with first- and multiple-episode schizophrenia spectrum disorders treated with quetiapine immediate release (ACCESS trial). J Clin Psychiatry 2010; 71: 1313 - 1323

20. Karow A, Reimer J, König $\mathrm{HH}$ et al. Cost-effectiveness of 12-month therapeutic assertive community treatment as part of integrated care versus standard care in patients with schizophrenia treated with quetiapine immediate release (ACCESS trial). J Clin Psychiatry 2012; 73: e402 - e408

21. Kissling W, Vogel C. Erfahrungen mit dem «Münchner Modell». Integrierte Versorgung - eine Zwischenbilanz. Neurotransmitter 2007; Sonderheft 2; 11 - 19

22. Roick C, Deister A, Zeichner D et al. Das Regionale Psychiatriebudget: Ein neuer Ansatz zur effizienten Verknüpfung ambulanter und stationärer Versorgungsleistungen. Psychiatr Prax 2005; 32: 177 - 184 
23. Roick C, Heinrich S, Deister A et al. Das Regionale Psychiatriebudget: Kosten und Effekte eines neuen sektorübergreifenden Finanzierungsmodells für die psychiatrische Versorgung. Psychiatr Prax 2008; 35: 279 - 285

24. Katon $\mathrm{W}$, Robinson $\mathrm{P}$, Von Korff $\mathrm{M}$ et al. A multifaceted intervention to improve treatment of depression in primary care. Arch Gen Psychiatry 1996; 53: 924 - 932

25. Clark RE, Teague GB, Ricketts SK et al. Cost-effectiveness of assertive community treatment versus standard case management for persons with co-occurring severe mental illness and substance use disorders. Health Serv Res 1998; 33 (5 Pt 1): 1285 $-1308$ 